\title{
Análise das Serologias para Infeções do Grupo TORCH e do Rastreio para Streptococcus do Grupo B na População de Grávidas de uma Maternidade
}

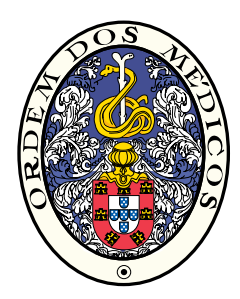

\author{
TORCH Serology and Group B Streptococcus Screening Analysis in the \\ Population of a Maternity
}

\author{
David LITO ${ }^{1}$, Telma FRANCISCO ${ }^{2}$, Inês SALVA ${ }^{2}$, Maria das Neves TAVARES ${ }^{3}$, Rosa OLIVEIRA ${ }^{4}$, Maria Teresa NETO ${ }^{5,6}$ \\ Acta Med Port 2013 Sep-Oct;26(5):549-554
}

\section{RESUMO}

Introdução: O rastreio para doenças de transmissão vertical na gravidez contribuiu para a melhoria dos cuidados perinatais. Objectivo: Avaliar o resultado de serologias para infeções do grupo TORCH e do rastreio para Streptococcus do grupo B (SGB) numa amostra de grávidas de uma maternidade, estudar a influência da idade e da nacionalidade, e identificar casos de infecção congénita. Material e Métodos: Estudo não probabilístico de prevalência de imunidade e infecção durante a gravidez.

Resultados: Registámos 9508 serologias TORCH e 2639 resultados de rastreio para SGB. A taxa de imunidade para rubéola fo $93,3 \%$, significativamente mais elevada em portuguesas; $25,7 \%$ das mulheres tinham IgG positiva para Toxoplasma goondii; a taxa foi mais elevada nas mulheres mais velhas e entre estrangeiras; encontrámos IgG positiva para vírus citomegálico humano (CMV) em 62,4\%; não houve variação com a idade. O VDRL foi reactivo em $0,5 \% ; 2,3 \%$ das mães tinham AgHBs positivo, mais frequente nas estrangeiras; $1,4 \%$ tinha anticorpos para o vírus da hepatite $\mathrm{C}$ e $0,7 \%$ tinha VIH positivo. Não houve casos declarados de infeção congénita; $13,9 \%$ das mulheres eram portadoras de SGB.

Discussão: A elevada taxa de imunidade para a rubéola é resultado da política nacional de vacinação. A baixa taxa de imunidade para a toxoplasmose torna mais dispendioso o seguimento das grávidas. A elevada prevalência do CMV está de acordo com o encontrado na comunidade. Para algumas infeções foram encontradas diferenças de acordo com a nacionalidade.

Conclusão: O conhecimento da imunidade e infecção na população é um instrumento importante para o planeamento dos rastreios durante a gravidez.

Palavras-chave: Complicações Infecciosas na Gravidez; Hepatite C; Infecções por Citomegalovirus; Infecções por Streptococcus; Rubéola; Toxoplasmose.

\section{ABSTRACT}

Introduction: Systematic screening for TORCH infections and group B Streptococcus (GBS) during pregnancy has been an important factor in the improvement of perinatal care.

Aim: To evaluate TORCH serology and GBS carriers state in the population of a maternity, to assess variability with age and nationality and to search for congenital infections.

Material and Methods: Non-probabilistic prevalence study.

Results: 9508 TORCH and 2639 GBS results were registered. Immunity rate for rubella was $93.3 \%$, higher for Portuguese women; for toxoplasmosis it was $25.7 \%$, higher among the oldest and foreign women; IgG for CMV was positive in $62.4 \%$, no influence of age was found. VDRL was reactive in $0.5 \%$; HBsAg was found to be positive in $2.3 \%$, higher in foreign women. Antibodies for hepatitis $\mathrm{C}$ virus and HIV were found respectively in $1.4 \%$ and $0.7 \%$. No congenital infections were diagnosed. GBS carrier state was found in $13.9 \%$. Discussion: A high rate of positive IgG was found for rubella reflecting vaccines policy. For toxoplasmosis the low rate of positives means that a high number of pregnant women have to repeat serology during pregnancy with inherent costs. Like in the general population, a high rate of CMV positive mothers was found. For some infections we found that foreign women had different conditions.

Conclusion: Knowledge on TORCH and GBS state helps to better draw guidelines concerning screening policies during pregnancy.

Keywords: Pregnancy Complications, Infectious; Cytomegalovirus Infections; Herpes Simplex; Hepatitis C; Streptococcal Infections; Rubella; Toxoplasmosis.

\section{INTRODUÇÃO}

A qualidade de cuidados prestados durante a gravidez, parto e período neonatal teve um enorme incremento em Portugal nas últimas décadas. Para além da regionalização dos cuidados perinatais, ${ }^{1,2}$ a sistematização dos exames a realizar durante a gravidez trouxe uma enorme mais-valia à grávida, ao feto e ao recém-nascido (RN).

As recomendações para o rastreio de infeções do gru- po TORCH eram, à data deste estudo, da responsabilidade da Divisão de Saúde Materna, Infantil e Adolescentes da Direcção-Geral da Saúde (DGS) do Ministério da Saúde e preconizava o rastreio para a rubéola, toxoplasmose, sífilis, hepatite $B(\mathrm{VHB})$ e vírus da imunodeficiência humana $(\mathrm{VIH}){ }^{3}$ No que respeita ao Streptococcus do Grupo B (SGB) verificou-se um aumento crescente do rastreio des-

1. Serviço de Pediatria e Neonatologia. Hospital de Vila Franca de Xira. Vila Franca de Xira. Portugal.

2. Serviço de Pediatria. Hospital Dona Estefânia. Lisboa. Portugal.

3. Unidade de Neonatologia do Hospital Dona Estefânia. Lisboa. Portugal.

4. Departamento das Ciências da Informação e Decisão em Saúde. CINTESIS. Faculdade de Medicina do Porto. Universidade do Porto. Porto. Portugal.

5. Unidade de Cuidados Intensivos Neonatais. Hospital Dona Estefânia. Lisboa. Portugal.

6. Faculdade de Ciências Médicas. Universidade Nova de Lisboa. Lisboa. Portugal.

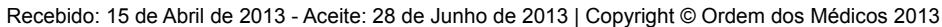


de 2002, em quase todas as maternidades, tornando-se quase generalizado mesmo antes de surgirem recomendações da DGS. ${ }^{4}$ Além disso, em situações de risco, é realizado o rastreio do anticorpo da hepatite $\mathrm{C}(\mathrm{VHC})$ e muitas grávidas são rastreadas para o vírus citomegálico humano (CMV) sem qualquer recomendação.

O Programa Nacional de Vacinação (PNV) inclui a vacina da rubéola para adolescentes do sexo feminino desde 1984, tendo-se tornado universal a partir de 1987, o que resulta numa taxa de imunidade muito elevada num grande grupo de mulheres em idade reprodutiva. Para a hepatite B a vacina integrou o PNV em 1995 para todos os adolescentes e, em 2000, teve início a vacinação universal dos $\mathrm{RN}$, crianças e adolescentes até aos 18 anos e outros indivíduos em risco. ${ }^{5} \mathrm{~A}$ divulgação das medidas de prevenção primária para a toxoplasmose durante a gravidez é uma rotina e, por fim, investiu-se muito na divulgação das vantagens do rastreio para o $\mathrm{VIH}$, nomeadamente durante a gravidez. $^{3}$

O segundo Inquérito Serológico Nacional realizado em 2002 pela DGS incluiu amostras populacionais de todos os grupos etários e indivíduos de ambos os géneros. ${ }^{6}$ Destinou-se a avaliar apenas o impacto do PNV na população em geral. Por isso parece-nos de grande interesse avaliar o estado imunológico das grávidas no que respeita a cada uma destas doenças.

À data do estudo a maternidade do Hospital Dona Estefânia estava integrada num hospital materno-infantil de Apoio Perinatal Diferenciadopor sua vez parte do Centro Hospitalar de Lisboa Central, EPE. Era uma maternidade de referência para gestações com diagnóstico pré-natal de anomalias congénitas com necessidade de intervenção cirúrgica no período neonatal. Os cerca de 2200 partos/ano provinham de uma área geográfica própria e de outras maternidades de Lisboa ou da zona Sul do País.

Um estudo recente de Ventura et al. caracteriza a população da maternidade no período de 2005 a $2008 .^{7}$

Foram objectivos deste estudo avaliar a prevalência de anticorpos séricos ou antigénios para o vírus da rubéola, Toxoplasma gondii, Treponema pallidum, VIH, VHB, CMV, VHC e estado de portador para o SGB, numa população de grávidas de uma maternidade, determinar taxas de seroconversão, e avaliar a influência da nacionalidade. Para a toxoplasmose e para o CMV estudou-se também a influência da idade na seropositividade.

\section{MATERIAL E MÉTODOS}

O presente estudo caracterizou-se como não probabilístico de prevalência de imunidade e infecção durante a gravidez para o vírus da rubéola, Toxoplasma gondii, Treponema pallidum, VIH, VHB, CMV, VHC e SGB.

A amostra foi não probabilística de conveniência. Recolhemos os resultados das serologias maternas dos processos clínicos dos RN no dia da alta hospitalar. Não foram incluídos resultados de serologias de mulheres cujas gestações foram interrompidas, independentemente do motivo da interrupção. A colheita de resultados ocorreu du- rante 22 meses de 2004 a 2006, 11 meses de 2006 a 2007 e dois meses em 2009. Registámos apenas um resultado para cada agente infeccioso - IgG positiva ou negativa correspondente ao terceiro trimestre da gestação, excepto quando detectada imunidade ou infeção num trimestre anterior; anotámos as seroconversões. Para a sífilis, rubéola, toxoplasmose e CMV registámos as infecções congénitas. $A$ taxa de seroconversão para a toxoplasmose e para o CMV calculou-se dividindo o número de mulheres com IgM positiva, pela soma de todas as mulheres com $\lg G$ negativa com as mulheres com IgM positiva, assumindo que todas as IgM positivas correspondiam a seroconversões recentes. Estudámos a influência da idade na taxa de seropositividade para toxoplasmose e CMV. Após a análise dos gráficos da distribuição para cada idade das mulheres imunes, efectuámos estudos para variados cutoffs etários de modo a estabelecer os grupos de idade com maior taxa de seropositivas. Os resultados são apresentados em percentagem com o respectivo intervalo de confiança $\left[\mathrm{IC}_{95 \%}\right]$. O teste exacto de Fisher, com um nível de significância de $5 \%(p<0,05)$ foi usado para estudar as diferenças de proporções entre nacionalidades. Para calcular a percentagem de vantagem nas mulheres mais velhas em relação às mais novas, calculámos o quociente de vantagem, Odds Ratio. Um Odds Ratio comum foi obtido pelo método não paramétrico de Mantel-Haensel Common Odds Ratio Estimate. Os dados foram analisados em SPSS, versão 19 para MacOS X.

\section{RESULTADOS}

Estudámos 12147 resultados de análises de 3162 muIheres, correspondendo a $26 \%$ do total de puérperas com alta da maternidade no período do registo. Dos 12147 resultados, 9508 eram serologias e 2639 produtos de zaragatoas rectais/vaginais para pesquisa de SGB. A mediana da idade materna foi 30 anos (mínimo 13 anos, máximo 48 anos); $28 \%$ das mulheres eram estrangeiras. Na Tabela 1 mostra-se a distribuição das nacionalidades e na Tabela 2 os registos de cada uma das serologias assim como a taxa de positivas/imunes.

Registámos 3104 serologias para a rubéola em 3104 mulheres. A taxa de mulheres imunes foi $93,3 \%[92,4$; $94,2]$, superior no grupo das portuguesas $-94,7 \%$ vs $89,7 \%$ $(p<0,001)$. Não encontrámos seroconversões na gravidez e não foi diagnosticado nenhum caso de rubéola congénita.

Para a toxoplasmose registámos 3126 resultados de 3126 mulheres; $25,7 \%$ [24,2; 27,2] tinham IgG positiva. Encontrámos maior taxa de positivas na população estrangeira $(30,6 \%$ vs $23,8 \%, p<0,001)$. Cinco mulheres $(0,16 \%)$ tinham tido IgM positiva durante a gravidez: em quatro a IgM era positiva de novo e numa era uma IgM de longa duração. A taxa de seroconversão estimada foi 1,7/1000 seronegativas. Em nenhum grupo etário a percentagem de mulheres com IgG positiva foi superior a $50 \%$. Nas mulheres com idade inferior a 30 anos a taxa de seropositividade foi $23 \%$ e nas mulheres com mais de 30 anos foi $30 \%$ $(p<0,05)$. Não foi diagnosticado nenhum caso de toxoplasmose congénita. 


\begin{tabular}{ccc}
\hline & No absoluto & \% do total \\
\hline Portugal & $\mathbf{2 2 7 7}$ & $\mathbf{7 2 , 0}$ \\
Estrangeiro & $\mathbf{8 8 5}$ & $\mathbf{2 8 , 0}$ \\
Brasil & 158 \\
PALOP & 91 & 2,0 \\
Europa & 94 & 3,0 \\
China & 56 & 1,8 \\
Outros países da Ásia & 55 & 1,7 \\
Outros países de África & 38 \\
América & 4 \\
Estrangeiro & 389 \\
\hline (país não conhecido)
\end{tabular}

Tabela 2 - Número de registos no estudo e resultados positivos (foi registado um resultado por mulher e por entidade estudada).

\begin{tabular}{|c|c|c|c|c|c|c|}
\hline \multicolumn{7}{|c|}{$\mathrm{N}^{\circ}$ de mulheres -3162} \\
\hline & \multicolumn{2}{|c|}{$\mathrm{N}^{\circ}$ de registos } & \multicolumn{3}{|c|}{ IgG positiva ou rastreio positivo } & \multirow[b]{2}{*}{$p$} \\
\hline & & Subtotal & $n(\%)$ & $\begin{array}{c}\text { Nacionais } \\
\%\end{array}$ & $\begin{array}{c}\text { Estrangeiras } \\
\%\end{array}$ & \\
\hline Rubéola (IgG) & 3104 & & $2896(93,3)$ & 94,7 & 89,7 & $<0,001$ \\
\hline Toxoplasmoses (lgG) & 3126 & & $804(25,7)$ & 23,8 & 30,6 & $<0,001$ \\
\hline CMV (IgG) & 640 & & $399(62,4)$ & 66,1 & 61,4 & 0,20 \\
\hline $\mathrm{VIH} 1$ e $2(\mathrm{Ac})$ & 567 & 9508 & $4(0,7)$ & 0,5 & 1,6 & 0,22 \\
\hline AgHBs & 576 & & $13(2,3)$ & 0,7 & 7,4 & $<0,001$ \\
\hline VDRL & 561 & & $3(0,5)$ & 0,5 & 0,8 & 0,54 \\
\hline $\mathrm{VHC}(\mathrm{Ac})$ & 934 & & $13(1,4)$ & 1,7 & 0,5 & 0,32 \\
\hline SGB & 2639 & 2639 & $368(13,9)$ & 13,4 & 15,5 & 0,18 \\
\hline Total & & 12147 & & & & \\
\hline
\end{tabular}

Registámos 640 resultados de serologia para CMV em 640 mulheres; $62,4 \%$ [58,6; 66,2] tinham IgG positiva; não foi encontrada diferença entre mulheres portuguesas e estrangeiras $(66,1 \%$ vs $61,4 \%, p=0,20)$. Oito mulheres $(1,3 \%)$ tinham IgG e IgM positivas; uma mulher tinha apenas IgM positiva. Taxa de seroconversão estimada - 0,4 a $3,6 / 1000$ seronegativas. Não houve variação na taxa de mulheres com IgG positiva com a idade. Não diagnosticámos nenhuma infecção congénita.
Recolhemos 561 resultados de VDRL em 561 mulheres, tendo sido reactivo em três - $0,5 \%[0,15 ; 18,87]$; duas mulheres eram portuguesas e uma estrangeira. O FTA-ABS foi negativo nas três. Não diagnosticámos casos de sífilis congénita.

Foi coligido o resultado do AgHBs de 576 mulheres e era positivo em $13(2,3 \%),[1,1 ; 3,5]$. A taxa de positividade foi $0,7 \%$ nas mulheres portuguesas e $7,4 \%$ nas estrangeiras $(p<0,001)$. A maior percentagem relativa de positivas 
foi encontrada nas mulheres de nacionalidade chinesa (três mulheres em nove) e nas da Guiné-Bissau (duas mulheres em sete).

$\mathrm{O}$ resultado do estudo do anticorpo para o $\mathrm{VHC}$ foi registado em 934 mulheres e era positivo em 13 (1,4\%) [0,7; $2,2]$ - em $1,7 \%[0,9 ; 2,5]$ das mulheres portuguesas e em $0,5 \%[0,1 ; 0,9]$ das estrangeiras (diferença não significativa, $p=0,32)$.

Das 567 mulheres em que foi registado o resultado do $\mathrm{VIH}$, encontrámos quatro resultados positivos $(0,7 \%)[1,4$ $4,3]$; duas mulheres eram portuguesas $(0,5 \%$ das mulheres portuguesas) e duas eram estrangeiras, ambas de Guiné-Bissau (duas de sete com esta nacionalidade). Procurámos concomitância de outras infecções com o VIH mas não encontrámos - todas as mulheres VIH positivo eram imunes para a rubéola, três tinham IgG negativa para toxoplasmose e em todas o VDRL foi não reactivo.

Por último, registámos os resultados de rastreio para o SGB em 2639 mulheres; o resultado foi positivo em 13,9\% $[12,6 ; 15,9](n=368)$. Não houve diferença significativa entre mulheres portuguesas e estrangeiras $(13,4 \%$ versus $15,5 \%, p=0,18)$.

\section{DISCUSSÃO}

O rastreio das doenças infecciosas de transmissão vertical durante a gravidez é de extrema importância e, muitas vezes, o único meio disponível para suspeitar que um RN assintomático pode estar infectado. Em Portugal estão bem estabelecidos os exames a realizar durante a gravidez e a respectiva periodicidade. ${ }^{3}$ Contudo, apesar do rastreio ser universal, os resultados não são coligidos nem divulgados, o que leva a um desconhecimento importante neste campo. O único estudo realizado neste âmbito foi o segundo Inquérito Serológico Nacional, uma avaliação do PNV da DGS publicado em 2004. Esse estudo engloba uma população de todos os grupos etários e de ambos os sexos e, como seria de esperar, exclui muitas das infecções não susceptíveis de prevenção por vacina. ${ }^{6}$

O presente estudo começou pelo registo de resultados do rastreio para o $S G B$, da rubéola e da toxoplasmose e evoluiu depois para um registo mais alargado englobando também o VDRL, hepatites, VIH e CMV. Este facto justifica a grande diferença entre números de mulheres no registo, rastreadas para uma ou outra destas doenças. Considerámos a amostra populacional representativa da maternidade uma vez que a população avaliada corresponde a $26 \%$ da população admitida no período do estudo e as suas características são semelhantes às encontradas no único estudo de caracterização da população da maternidade de que temos conhecimento. ${ }^{7}$

A imunidade para a rubéola é elevada - 93,3\%. Apesar de ter utilizado metodologia diferente, um estudo realizado em 1988 nesta mesma maternidade encontrou uma taxa de imunidade de $82 \% .{ }^{8} \mathrm{~A}$ diferença é certamente devida à introdução da vacina no PNV em 1987, o que justifica também que a percentagem de mulheres susceptíveis ao vírus da rubéola seja menor na população portuguesa do que nas mulheres estrangeiras $-5,2$ vs $10,0 \%(p<0,001)$. No segundo Inquérito Serológico Nacional (2001 - 2002) a taxa variou entre $94,9 \%$ e $97,9 \%$ dependendo dos grupos etários. ${ }^{6}$ Este valor mais elevado pode ser devido precisamente ao facto de, nesse inquérito serem englobadas mulheres de todos os grupos etários enquanto que a população deste estudo incluiu apenas mulheres em idade reprodutiva. De 1997 a 2008, último ano de publicação do registo de Doenças de Declaração Obrigatória da DGS, não houve em Portugal casos declarados de rubéola congénita. ${ }^{9}$

Num estudo recente realizado em mulheres em idade reprodutiva no Norte de Portugal, a taxa de seropositividade para a toxoplasmose foi $24,4 \%{ }^{10}$. No nosso estudo foi semelhante $-25,7 \%$ mas, no estudo realizado em 1988 esta taxa foi $37,9 \% .{ }^{8} \mathrm{~A}$ taxa de imunes foi inferior nas mulheres portuguesas comparadas com as estrangeiras $-23,6 \%$ vs $30,0 \%, p<0,001$. Essa diferença foi ainda maior quando se comparou mulheres europeias com as dos países africanos de língua portuguesa e outros países africanos - 45,6\% e $46,2 \%$, respectivamente $(p<0,001)$. Em França, em 2003, a taxa de seropositividade em grávidas estimava-se em $43,8 \%$, independentemente da nacionalidade, sendo mais elevada que no resto dos países europeus. ${ }^{11}$ Num estudo realizado em Espanha entre 2006 e 2010, ${ }^{12}$ a imunidade para a toxoplasmose foi significativamente superior em grávidas imigrantes quando comparadas com as espanholas, $41,4 \%$ vs $12 \%$, $(p<0,001)$. Nesse estudo, tal como no nosso, verificou-se que a seropositividade aumentava com a idade. No que respeita à taxa de seroconversão atendendo a que, das cinco mulheres com lgM positiva quatro tinham IgM positiva de novo e, uma, IgM de longa duração, parece poder estimar-se que a taxa de seroconversão rondou os $1,7 / 1000$ seronegativas.

Desde 2006 está indicado o estudo da serologia para o CMV em consulta pré-concepcional. Contudo, nos últimos anos, muitas mulheres têm feito a análise durante a gravidez quando decidem fazer colheita de sangue do cordão para células estaminais. Neste estudo a taxa de seropositividade para o CMV foi $62,4 \%$, muito inferior aos $85 \%$ encontrados no estudo de 1988 realizado na mesma maternidade. $^{8}$ No segundo Inquérito Serológico Nacional, das 595 mulheres com idade entre os 15 e os 64 anos, a taxa de seropositivas variou entre 71,3 e $92,2 \% .{ }^{6}$ Num estudo realizado em Portugal numa população de grávidas, a taxa de positivas calculada situava-se entre os $72,2 \%$ e os $94,1 \%$. Nesse mesmo estudo foi encontrada uma taxa de infecção congénita de $0,7 \%$ a $1 \%$ dos nados-vivos. ${ }^{13}$ No presente estudo não foi diagnosticada nenhuma infecção congénita. Tal como no estudo de $1988,{ }^{8}$ atrás referido, não se encontrou uma maior taxa de seropositividade nas mulheres mais velhas. Pelo contrário, num estudo realizado em Espanha em 1999 os autores encontraram maior seropositividade em mulheres de mais idade. ${ }^{14}$ Sem rastreio pré-concepcional para CMV é muito difícil determinar a taxa de seroconversão durante a gravidez. O diagnóstico de seroconversão pode erradamente ser feito baseado numa IgM positiva que afinal era apenas uma lgM de longa duração. No nosso 
estudo oito mulheres tinham IgM e IgG positivas não se conhecendo desde quando, e uma tinha apenas IgM positiva. Por isso, ao contrário do que aconteceu com a toxoplasmose, a estimativa de taxa de seroconversão tem valores tão díspares, podendo considerar-se como certo qualquer valor entre 0,4 a 3,6/1000 seronegativas.

Neste estudo a taxa de mulheres com VDRL reactivo foi $0,24 \%$. Em nenhum caso as provas treponémicas eram positivas. Dado o reduzido número de casos, não foi possível avaliar prevalências de acordo com a nacionalidade. Apesar de a sífilis dever ser rastreada na gravidez, ter diagnóstico fácil e terapêutica específica, continua a existir sífilis congénita em Portugal, embora o número de casos declarados tenha vindo a diminuir progressivamente em Portugal. ${ }^{15}$ É por isso preocupante que as novas Normas de Orientação Clínica da DGS/OM tenham suprimido o pedido de VDRL no segundo trimestre nas gestações de baixo risco, sabendo nós que o risco para sífilis na gravidez não conhece fronteiras sociais ou económicas.

A taxa de mulheres AgHBs positivas foi 2,3\%. Noutra série, de 779 grávidas nesta mesma maternidade no ano de 1990 , foi $2,7 \%$ e, num estudo realizado no Norte de Portugal em 1993/4, em 627 grávidas a taxa de positivas foi 2,9\%; ${ }^{16,17}$ no $2^{\circ}$ Inquérito Serológico Nacional, de 1095 indivíduos com idades entre dois e mais de 65 anos, a taxa de positivos foi $0,36 \% .{ }^{6}$ Neste estudo, as mulheres estrangeiras tiveram uma taxa de seropositividade muito superior $-7,4 \%$ vs $0,8 \%, p<0,001$. Este achado pode dever-se, mais uma vez, à política de vacinação, uma vez que muitas mulheres portuguesas em idade reprodutiva já fazem parte do grupo que beneficiou da vacina na adolescência.

Em Portugal estima-se que a prevalência do anticorpo da hepatite C seja 1,5\%. ${ }^{18,19} \mathrm{Na}$ gravidez a serologia só é pedida nos grupos considerados de risco. ${ }^{19}$ Neste estudo a taxa de seropositividade foi $1,4 \%$. Não foi encontrada diferença significativa entre grávidas portuguesas e estrangeiras.

$\mathrm{Na}$ Europa, a taxa de positivos para o $\mathrm{VIH}$ manteve-se estável nos últimos anos, variando entre 6,5 em 2004 e 5,7 em 2009 por 100000 habitantes. ${ }^{20}$ A taxa em Portugal continua a ser elevada - 2,8 por 100000 habitantes em 2009. ${ }^{21}$ A taxa de seropositivas foi muito superior à encontrada noutros estudos europeus. Na Holanda, Eline Coul refere taxas de $0,04 \%$ a $0,08 \%$ em 2008 e numa população da Catalunha, Carnicer-Pont refere taxas de $0,17 \%$ em 2009. ${ }^{22,23}$ Não foram encontradas co-infecções nas mulheres VIH positivas.

\section{REFERÊNCIAS}

1. Niebler A. EU benchmarking report 2009-2010. Munich: European Foundation for the Care of Newborn Infants; 2010.

2. Neto MT. Perinatal care in Portugal: Effect of 15 years of a regionalized system. Acta Paediatr. 2006;95:1349-52.

3. Direcção-Geral da Saúde. Saúde reprodutiva: Doenças infecciosas e gravidez. Orientações técnicas 11. Lisboa: DGS; 2000.

4. Neto MT. Group B streptococcal disease in Portuguese infants younger than 90 days. Arch Dis Child Fetal Neonatal Ed. 2008;93:F90-3.

5. Direcção-Geral da Saúde. Programa Nacional de Vacinação. Orientações Técnicas 10. Lisboa: DGS; 2006.

6. Direcção-Geral da Saúde. Avaliação do Programa Nacional de Vaci-
O SGB é o agente mais frequente de infecção bacteriana de transmissão vertical. ${ }^{4}$ Em 2004 publicaram-se em Portugal as primeiras recomendações para a prevenção da transmissão vertical do SGB. ${ }^{24}$ Num estudo realizado entre 2001 e 2005 em Portugal, a incidência global de sépsis comprovada por SGB nos três primeiros meses de vida foi 0,54/1 $000[0,47 ; 0,60]$ nados-vivos. ${ }^{4}$ Este estudo foi continuado por mais dois anos. Ao longo dos seis anos verificou-se diminuição de infecção confirmada de 0,6/1000 nados-vivos em 2002 para 0,15/1 000 nados-vivos em 2007.4,25 O rastreio do estado de portadora para o SGB e a profilaxia da transmissão vertical modificaram o panorama das mortes neonatais por infecção bacteriana de transmissão vertical. ${ }^{4,26}$ Em 2005, um estudo do Norte de Portugal identificou uma taxa de colonização de $34,9 \%$ em grávidas. ${ }^{27}$ Nesta mesma maternidade, num estudo realizado entre 2007 e 2008 englobando $85 \%$ da população de grávidas, 18\% estavam colonizadas. ${ }^{28}$

O conhecimento da prevalência das infecções susceptíveis de transmissão vertical na população de grávidas de uma região ou país pode ajudar a definir que rastreios devem ser incluídos num programa de seguimento da gravidez. A distinção entre mulheres de nacionalidade portuguesa e estrangeiras, nesta área, pode acrescentar informação e resultar em acções de sensibilização, tanto para o rastreio como para a vacinação da população de imigrantes contra infecções virtualmente erradicadas da população portuguesa.

\section{CONCLUSÕES}

Apesar de se tratar da população de uma única maternidade, o elevado número de mulheres envolvidas e de resultados analisados gerou informação útil. Estes dados podem questionar a pertinência do rastreio universal para determinados agentes infecciosos. A nacionalidade, nomeadamente, a distinção entre mulheres nativas e imigrantes implicou diferentes prevalências na imunidade para rubéola e toxoplasmose bem como na infecção pelo vírus da hepatite $B$.

\section{CONFLITO DE INTERESSES}

Nada a declarar.

\section{FONTES DE FINANCIAMENTO}

Os autores negam ter beneficiado de qualquer subsídio ou bolsa que tenham contribuído para a realização do trabalho.

nação - $2^{\circ}$ Inquérito Serológico Nacional - Portugal Continental 20012002. Lisboa: DGS; 2004.

7. Ventura MT, Gomes MC. Análise descritiva de parturientes e recém-nascidos na maternidade. Hospital Dona Estefânia entre 2005 e 2008. Acta Med Port. 2010;23:793-802.

8. Neto MT, Borges D, Amaral JV. As infecções do grupo TORCH. Rev Port Pediatr. 1992;23:71-7.

9. Direcção Geral da Saúde, Direcção de Serviços de Informação e Análise - Divisão de Epidemiologia. Doenças de Declaração Obrigatória, 1997-2001, 2001 - 2005, 2005-2008. Lisboa: DGS; 2006.

10. Lopes AP, Dubey JP, Moutinho O, Gargaté MJ, Vilares A, Rodrigues 
M, et al. Seroepidemiology of Toxoplasma gondii infeccion in women from North of Portugal in their childbearing years. Epidemiol Infect. 2011;31:1-6.

11. Berger F, Goulet V, Le Strat Y, Desenclos JC. Toxoplasmosis among pregnant women in France: risk factors and change of prevalence between 1995 and 2003. Rev Epidemiol Sante Publique. 2009;57:241-8.

12. Ramos JM, Milla A, Rodríguez JC, Padilla S, Masiá M, Gutiérrez. F. Seroprevalence of toxoplasma gondii infection among immigrant and native pregnant women in Eastern Spain. Parasitol Res. 2011;109:144752.

13. Lopo S, Vinagre E, Palminha P, Paixão MT, Nogueira P, Freitas MG. Seroprevalence to cytomegalovirus in the Portuguese population, 20022003. Euro Surveill. 2011;16:pii=19896.

14. Ory F, Ramírez R, Comas LG, Leon P, Sagües MJ, Sanz JC. Is there a change in cytomegalovirus seroepidemiology in Spain? Eur J Epidemiol. 2004;19:85-9.

15. Neto MT. Syphilis: Still and once more. In: Pereira-da-Silva L, Neto MT, editors. New Challenges in Foetal and Neonatal Infections. Kerala: Research Signpost; 2011. p.157-66.

16. Neto MT, Ventosa L, Loureiro V, Leal F, Henriques M, Amaral JM. Transmissão vertical do vírus da hepatite $\mathrm{B}$. Importância do rastreio na grávida. Rev Port Ped. 1992;23:79-84.

17. Lima ML, Rodrigues G, Gonçalves H, Lino C, Carrapatoso C, Nogueira R. Rastreio do antigénio HBs na população obstétrica da maternidade de Júlio Dinis. Rev Port Clin Geral. 2000;16:35-42.

18. Marinho RT, Moura MC, Giria JA, Ferrinho P. Epidemiological aspects of hepatitis C in Portugal. J Gastroenterol Hepatol. 2001;16:1076-77.

19. Bacalhau S, Timóteo C, Agro J. Transmissão perinatal do vírus da Hepatite C - Hospital Santo André, Leiria 2002-2006. Acta Med Port.
2010:23:391-8

20. Likatavicius G, Van de Laar MJ. HIV infection and AIDS in the European Union and European Economic Area, 2010. Euro Surveill. 2011;16(48):pii=20030.

21. Likatavicius G, Van de Laar MJ. HIV and AIDS in the European Union, 2009. Euro Surveill. 2010;15(48):pii=19737. [Consultado 2012 Jun 1].

22. Op de Coul EL, Hahné S, Weert YW, Oomen P, Smit C, Ploeg KP, et al Antenatal screening for HIV, hepatitis $B$ and syphilis in the Netherlands is effective. BMC Infect Dis. 2011;11:185.

23. Carnicer-Pont D, Almeda J, Marin JL, Martinez C, Gonzalez-Soler MV, Montiliu A, et al. The HIV NADO working Group. Unlinked anonymous testing to estimate HIV prevalence among pregnant women in Catalonia, Spain, 1994 to 2009. Euro Surveill. 2001;16(32):pii=19940.

24. Almeida A, Agro J, Ferreira L. Estreptococo b Hemolítico do Grupo B. Protocolo de rastreio e prevenção da doença perinatal. Secção de Neonatologia da Sociedade Portuguesa de Pediatria. Consensos Nacionais em Neonatologia. Setúbal: Angelini Farmacêutica; 2004.

25. Neto MT. GBS screening and perinatal infection: controversial aspects. J Matern Fetal Neonatal Med. 2010;23:S56

26. Van Dyke MK, Phares CR, Lynfield R, Thomas AR, Arnold KE, Craig AS et al. Evaluation of universal antenatal screening for group B Streptococcus. N Engl J Med. 2009;360:2626-36.

27. Areal A, Moreira M, Nunes S, Faustino MA, Cardoso L, Sá C. Determinantes da colonização materna e da infecção neonatal por Streptococcus do grupo B. Acta Obstet Ginecol Port. 2008;2:72-9.

28. Mascarenhas A, Rodrigues V, Constantino C, Nóbrega S, Virella D, Neto MT. Group B Streptococcus screening: repercussion in the postnatal ward dynamics. J Matern Fetal Neonatal Med. 2010;23:S390. 